
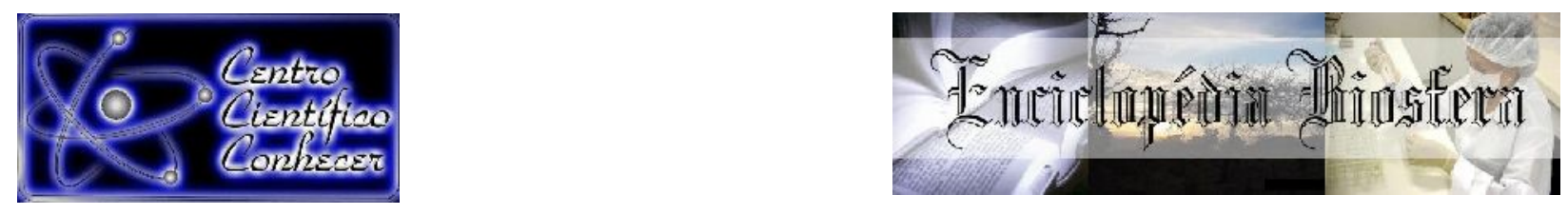

\title{
VARIAÇÃO ESPAÇO-TEMPORAL E CORRELATOS DAS VARIÁVEIS LIMNOLÓGICAS DO RIO XINGU A MONTANTE DA USINA HIDRELÉTRICA DE BELO MONTE, ALTAMIRA-PA
}

\author{
${ }^{1}$ Ana Caroline Leal Nascimento; ${ }^{2}$ Kleiton Rabelo de Araújo; ${ }^{3}$ Karina Dias-Silva \\ 1Programa de Pós-graduação em Biodiversidade, Universidade Federal do Oeste do \\ Pará (UFOPA), Santarém-PA, Brasil. \\ 1carolineleal.bio@gmail.com \\ 2 Programa de Pós-Graduação em Geoquímica e Geotectônica, Universidade de \\ São Paulo (USP), São Paulo-SP, Brasil. \\ ${ }^{3}$ Programa de Pós-Graduação em Biodiversidade e Conservação, Universidade \\ Federal do Pará (UFPA), Altamira-PA, Brasil.
}

Recebido em: 15/05/2021 - Aprovado em: 15/06/2021 - Publicado em: 30/06/2021 DOI: 10.18677/EnciBio 2021B24

\begin{abstract}
O Xingu é um dos rios principais que compõem a bacia Amazônica e possui diversos usos como abastecimento, transporte, agricultura, geração de energia, subsistência de comunidade tradicionais, recreação e lazer. O monitoramento de variáveis limnológicas em ecossistemas aquáticos é significativo por funcionar como indicador das condições de corpos d'água. Diante disso, este trabalho teve como objetivo analisar e caracterizar a variação espaço-temporal das variáveis limnológicas a montante da UHE Belo Monte na cidade de Altamira-PA. Foram medidas as variáveis Temperatura da água $\left({ }^{\circ} \mathrm{C}\right)$, Oxigênio Dissolvido (OD) $(\mathrm{mg} / \mathrm{L}), \mathrm{pH}$, Condutividade Elétrica $(\mu \mathrm{S} / \mathrm{cm})$ e Turbidez (NTU), pré e pós-barramento, nos períodos seco e chuvoso. Foi realizada uma análise de componentes principais (PCA) para todas as variáveis físico-químicas, como análise exploratória dos dados. Para os dados pós-represamento, utilizamos ANOVA para testar a variação espacial e temporal entre as variáveis. Com a finalidade de analisar a variação entre os períodos (seco e chuvoso) foi feito um Teste $T$ de variâncias separadas. Por fim, para determinar a correlação das variáveis, aplicamos a correlação de Pearson. Não foi observado diferenças espaciais e temporais entre as variáveis analisadas. Contudo, em relação ao período, a temperatura da água apresentou maior média durante o período seco. Enquanto Turbidez e $\mathrm{pH}$ apresentaram maior média durante o período chuvoso. Desta forma, a instalação da usina no rio Xingu promoveu alterações nas características limnológicas quando considera-se a sazonalidade.
\end{abstract}

RESUMO

PALAVRAS-CHAVE: Análise espaço-temporal; Qualidade de água; sazonalidade. 


\title{
SPATIO-TEMPORAL VARIATION AND CORRELATES OF LIMNOLOGICAL VARIABLES OF THE XINGU RIVER UPSTREAM OF THE BELO MONTE HYDROELECTRIC PLANT, ALTAMIRA-PA
}

\begin{abstract}
The Xingu is one of the mains rivers compounding the Amazonian Watershed and it has several uses such as supply, transportation, agriculture, power generation, traditional community subsistence, recreation and leisure. The monitoring of limnological variables in aquatic ecosystems is significant by its role as an indicator of water bodies conditions. Therefore, this study aimed to analyze and characterize the spatio-temporal variation of the limnological variables upstream of the Belo Monte hydropower Plant in the city of Altamira-PA. Water temperature $\left({ }^{\circ} \mathrm{C}\right)$, Dissolved Oxygen (DO) (mg / L), $\mathrm{pH}$, Electrical Conductivity $(\mu \mathrm{S} / \mathrm{cm})$ and Turbidity (NTU) variables were measured, pre and post-impounding in the dry and wet periods. We performed a principal component analysis (PCA) for all physical-chemical variables, as an exploratory analysis of the pre-damming data. For pre-impoundment data, we used ANOVA to test the spatio-temporal variation between variables. In order to analyze the variation between periods (dry and wet), a $T$ test of separate variances was performed. Finally, to determine the correlation of the variables, we apply Pearson's correlation. There was no difference in the spatio-temporal variation between the variables analyzed. However, in relation to the period, the water temperature showed a higher average during the dry period. While Turbidity and $\mathrm{pH}$ showed highest average during the wet season. Thus, the installation of the plant on the Xingu River promoted changes in limnological characteristics when considering seasonality.
\end{abstract}

KEYWORDS: Spatio-temporal analysis; Water quality; seasonality.

\section{INTRODUÇÃO}

Nas últimas décadas houve um aumento no número de construção de grandes barragens para geração de energia elétrica (MULLIGAN et al., 2020), devido ao crescimento pela demanda por alimento e energia (St. LOUIS et al., 2000). Rios, mesmo de grande extensão, possuem sensibilidade pronunciada a alterações no uso da terra que podem ser refletidas nas propriedades de suas águas (BATALHA et al., 2014; VOZA et al., 2015). Na Amazônia oriental, mudanças no uso da terra (I.E. desmatamento, agricultura, mineração, agropecuária, empreendimentos hidrelétricos) ganharam incentivo econômico e político na década de 1970 com a construção da rodovia Transamazônica (SKOLE et al., 1994; LAURANCE, 2001; NEPSTAD et al., 2014).

O rio Xingu é um dos inúmeros rios amazônicos com extenso histórico de atividades que causam mudanças no uso da terra em sua bacia (SILVÉRIO et al., 2015). É o maior tributário de águas claras do rio Amazonas e fonte de subsistência e lazer para diversas comunidades tradicionais e cidades de seu entorno, junto com os rios Tapajós e Tocantins drenam mais de $50 \%$ do Território paraense (SANTOS et al., 2016). A partir de 2011 foram iniciadas as obras para a implantação da Usina Hidrelétrica (UHE) de Belo Monte, na região da Volta Grande do Xingu, finalizada no ano de 2019. A transformação de ambientes lóticos em lênticos, pela construção de barragens, resulta em mudanças no regime hidrológico, na qualidade da água e nas condições ecológicas, as quais podem causar mudanças nas comunidades aquáticas, nas variáveis limnológicas e no ecossistema como um todo (PELICICE et al., 2015; CASTELLO; MACEDO, 2016). 
Durante os primeiros anos após o represamento podem surgir alterações substanciais na qualidade da água e nas variáveis físico-químicas do rio (CHANUDET et al., 2016). Rios com represas podem apresentar desde variações na temperatura da água, oxigênio dissolvido, acúmulo de sedimento, concentração de nutrientes até turbidez (POFF; WARD, 1989; TRÉPANIER et al., 1996; AGOSTINHO et al., 2008; SHELDON et al., 2012; ALMEIDA et al., 2019; NOGUEIRA et al., 2021). Portanto, alterações nessas variáveis podem ser uma resposta à impactos de atividades humanas como flutuações em valores de $\mathrm{pH}$ e oxigênio dissolvido (RíOSVILLAMIZAR et al., 2017), levando em consideração a sazonalidade, já que existe um potencial de incremento nas flutuações de variáveis físico-químicas, devido aos efeitos da sazonalidade amazônica.

Belo Monte apresenta uma estrutura de barragem a fio d'água, diferente da maioria das hidrelétricas brasileiras. Embora impactos desses tipos de barragens sejam considerados frequentemente menores, isso tem sido questionado para projetos em grandes rios ao redor do mundo (EGRÉ; MILEWSKI, 2002; ANDERSON et al., 2015). Um agravante é a escassez de informação na literatura quanto a descrição de variáveis físico-químicas e suas alterações nestes ambientes. Geralmente estudos avaliando essas variáveis em reservatórios hidrelétricos não possuem informação posterior ao represamento (CHANUDET et al., 2016; LING et al., 2017; CONCEIÇÃO et al., 2021). Dessa forma, se tornam necessários estudos sobre as condições da água pré e pós-barramento, visando entender do estado atual do rio e gerar conhecimentos aprofundados acerca das condições hidrológicas (TUNDISI, 2007).

Devido os riscos que a deterioração da qualidade da água pode causar, à saúde e bem-estar humanos e de outros organismos que dependem dela para sobreviver, a qualidade da água dos rios no Brasil deve obedecer a parâmetros físico-químicos mínimos previstos pela resolução № 357 do Conselho Nacional do Meio Ambiente - CONAMA (CONAMA, 2005). Finalmente, o monitoramento de variáveis limnológicas é necessário para garantir o funcionamento de ecossistemas e condições favoráveis à subsistência humana. Com base nisso, este trabalho teve como objetivo analisar e caracterizar a variação espaço-temporal das variáveis limnológicas a montante da UHE Belo Monte na cidade de Altamira-PA.

\section{Área de estudo}

\section{MATERIAL E MÉTODOS}

À margem esquerda do rio Xingu, está localizado o município de Altamira que possui área de $161.445,93 \mathrm{~km}^{2}$ e população de 94.624 pessoas (IBGE, 2010) na microrregião do Sudoeste do Pará. O Xingu é um rio de águas claras e antes do represamento apresentava $\mathrm{pH}$ em torno de 6,0 a 7,0, com pouco sedimento em suspensão e consequentemente zona fótica pronunciada (SIOLI, 1984). Segundo a classificação de Koppen (1984) a região apresenta clima tropical, do tipo Am com sazonalidade bem definida em dois períodos. O período seco ocorre de junho a novembro e o chuvoso de dezembro a maio, com temperatura e pluviosidade média anual de $26,2{ }^{\circ} \mathrm{C}$ e $144 \mathrm{~mm}$, respectivamente (INMET, 2015). Localizada a aproximadamente $50 \mathrm{~km}$ do município de Altamira foi instalada a UHE de Belo Monte. Dentre os impactos da construção, está a área inundada de aproximadamente $518 \mathrm{~km}^{2}$ que corresponde principalmente às áreas de margem e terra firme (ELETROBRÁS, 2009). 


\section{Delineamento amostral}

No total, foram amostrados oito pontos de coleta, sendo quatro em praias destinadas a atividades de recreação às margens do rio Xingu (Figura 1), com base nas coordenadas $03^{\circ} 12^{\prime} \mathrm{S}$ e $52^{\circ} 12^{\prime}$ 'W. As amostragens ocorreram mensalmente durante um ano, de setembro de 2018 a setembro de 2019. A análise temporal abrangeu os períodos: chuvoso e de seca, e cada praia foi comparada espacialmente. Em cada ponto amostral foram caracterizados: temperatura da água $\left({ }^{\circ} \mathrm{C}\right)$, oxigênio dissolvido $(\mathrm{OD})(\mathrm{mg} / \mathrm{L}), \mathrm{pH}$, condutividade elétrica $(\mu \mathrm{S} / \mathrm{cm})$ e turbidez (NTU), com auxílio de medidores portáteis de campo (Hanna HI98129, Instrutherm MO-910 e QUIMIS Q279P, respectivamente).

FIGURA 1 - Localização da área de estudo. Os pontos amostrais são representados em vermelhos, com localizações em PR1 a PR4 agrupando dados deste trabalho. Já $A M B$ 02, PAN 02, RX 01, RX 02, RX 18 e $R X 24$ representam dados do monitoramento da Norte Energia.

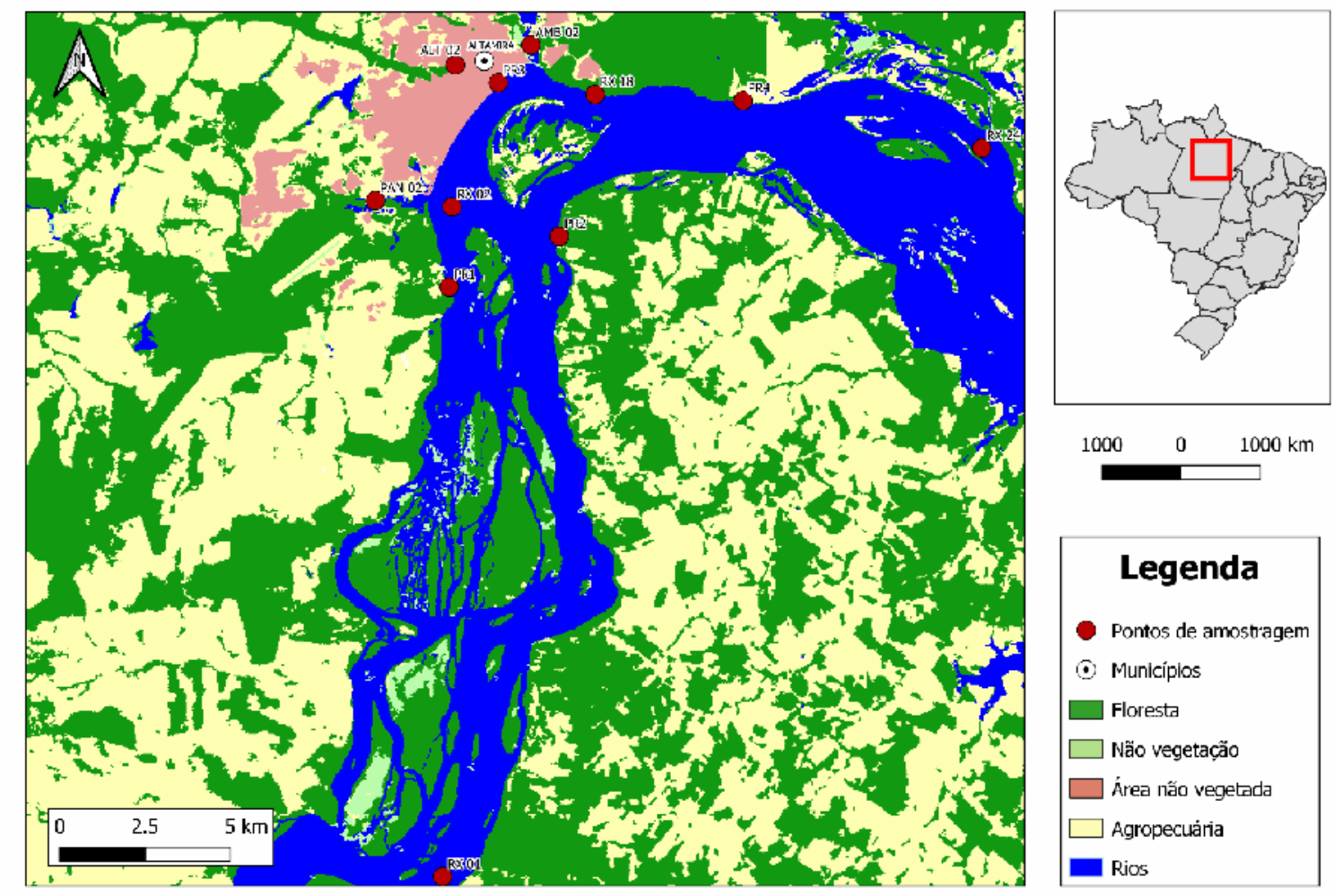

Fonte: Autores

Dentre as praias amostradas, PR 02, PR 03 e PR 04 também fazem parte do monitoramento desenvolvido pela Norte Energia, empresa responsável pela UHE Belo Monte. Com base nos dados do monitoramento da Norte Energia, foi realizado uma série histórica de dez anos (2010 a 2020) da área amostrada antes do represamento do rio Xingu. Para isso, foram selecionados quatro pontos $\mathrm{RX} 01, \mathrm{RX}$ $02, R X 18$ e $R X 24$ devido à proximidade com as praias monitoradas por este trabalho. Foram selecionadas as mesmas variáveis físico-químicas: Temperatura da água, oxigênio dissolvido (OD), pH, condutividade elétrica e turbidez. 


\section{Análises estatísticas}

Foi realizada uma análise de componentes principais (PCA) para todas as variáveis físico-químicas, como análise exploratória dos dados pré-represamento (LEGENDRE; LEGENDRE, 2012). Para os dados pós-represamento foi utilizada uma análise de variância (ANOVA dois fatores) para testar a variação espacial entre as variáveis físico-químicas, obedecendo os pressupostos de normalidade e de homogeneidade (ZAR, 2010). Já a variação das médias por período foi testada com um Teste $T$ de variâncias homogêneas quando os dados cumpriram os pressupostos, e um Teste $T$ para variâncias separadas quando os pressupostos não foram cumpridos (ZAR, 2010). Para determinar a correlação das variáveis, foi utilizada a correlação de Pearson (ZAR, 2010). Todos os testes foram realizados no ambiente $R$ Statistics utilizando os pacotes adequados (I.E. vegan, car) (R CORE TEAM, 2019; OKSANEN et al., 2020).

\section{RESULTADOS E DISCUSSÃO}

As concentrações das variáveis físico-químicas registraram padrões seguindo a influência do período sazonal. A máxima de OD durante o estudo foi de $8,3 \mathrm{mg} \mathrm{L}^{-1}$, registrada no período chuvoso, já a mínima foi de $6,2 \mathrm{mg} \mathrm{L}^{-1}$ durante o período seco. $\mathrm{O}$ pH também seguiu este padrão com máxima de 8,22 e mínima de 5,3 durante o período chuvoso e seco, respectivamente. De outra forma, temperatura da água apresentou máxima de $32,5^{\circ} \mathrm{C}$ durante o período seco e mínima de $28,3{ }^{\circ} \mathrm{C}$ no período chuvoso. O mesmo padrão foi observado para a condutividade elétrica com máxima de $31 \mu \mathrm{S} \mathrm{cm} \mathrm{cm}^{-1}$ e mínima de $11 \mu \mathrm{S} \mathrm{cm} \mathrm{cm}^{-1}$ (Tabela 1).

TABELA 1 - Variáveis limnológicas (temperatura da água, Oxigênio Dissolvido, Potencial Hidrogeniônico $(\mathrm{pH})$, Condutividade Elétrica, Turbidez) analisadas durante os períodos chuvoso e seco. São apresentadas médias de cada variável referente a todo o período estudado na linha em negrito (total). $\mathbf{D P}=$ Desvio padrão.

\begin{tabular}{lrrrr}
\hline Variáveis & Média & \multicolumn{1}{c}{ DP } & Mínimo & Máximo \\
\hline Temperatura período chuvoso & 29,57 & 0,91 & 28,3 & 31,20 \\
Temperatura período seco & 31,5 & 0,53 & 30,7 & 32,50 \\
Temperatura total & $\mathbf{3 0 , 2 1}$ & $\mathbf{1 , 2 2}$ & $\mathbf{2 8 , 3}$ & $\mathbf{3 2 , 5}$ \\
Oxigênio Dissolvido período chuvoso & 6,67 & 0,72 & 5,6 & 8,30 \\
Oxigênio Dissolvido período seco & 6,38 & 0,21 & 6,2 & 6,70 \\
Oxigênio Dissolvido total & $\mathbf{6 , 5 7}$ & $\mathbf{0 , 6 1}$ & $\mathbf{5 , 6}$ & $\mathbf{8 , 3 0}$ \\
pH período chuvoso & 6,74 & 0,56 & 6,11 & 8,22 \\
pH período seco & 5,88 & 0,44 & 5,3 & 6,50 \\
pH total & $\mathbf{6 , 4 5}$ & $\mathbf{0 , 6 6}$ & $\mathbf{5 , 3}$ & $\mathbf{8 , 2 2}$ \\
Condutividade período chuvoso & 17,31 & 4,84 & 11 & 27 \\
Condutividade período seco & 18,25 & 6,58 & 11 & 31 \\
Condutividade total & $\mathbf{1 7 , 6 2}$ & $\mathbf{5 , 3 5}$ & $\mathbf{1 1}$ & $\mathbf{3 1}$ \\
Turbidez período chuvoso & 12,76 & 6,29 & 4,87 & 30,4 \\
Turbidez período seco & 5,68 & 3,39 & 2,4 & 11 \\
Turbidez total & $\mathbf{1 0 , 4}$ & $\mathbf{6 , 3 9}$ & $\mathbf{2 , 4}$ & $\mathbf{3 0 , 4}$ \\
\hline
\end{tabular}


A variação espaço-temporal foi analisada, e não foi registrada diferença significativa entre as variáveis. No entanto, durante os períodos seco e chuvoso, as variáveis Temperatura da água, $\mathrm{pH}$ e Turbidez, apresentaram diferença (Figura 2). A Temperatura da água teve um aumento $1,93^{\circ} \mathrm{C}$ no período seco $(T=-6,509$, $\mathrm{p}=0,001)$. Enquanto o $\mathrm{pH}$ teve um aumento de 0,86 no período chuvoso $(\mathrm{T}=4,011$, $\mathrm{p}=0,001)$. De maneira similar, a turbidez teve aumento de 7,08 NTU também no período chuvoso $(T=3,579, p=0,001)$. As concentrações de $O D$ e condutividade elétrica, não sofreram alterações expressivas nos períodos analisados.

FIGURA 2: Comparação de mediana das variáveis nos períodos amostrados nas praias das margens do rio Xingu, Altamira-PA. A) Temperatura da água; B) $\mathrm{pH}$ da água e C) Turbidez da água. Linhas centrais correspondem à mediana, a caixa o primeiro e o terceiro quartil, as barras representam os desvios padrão e o ponto valores discrepantes.

A)

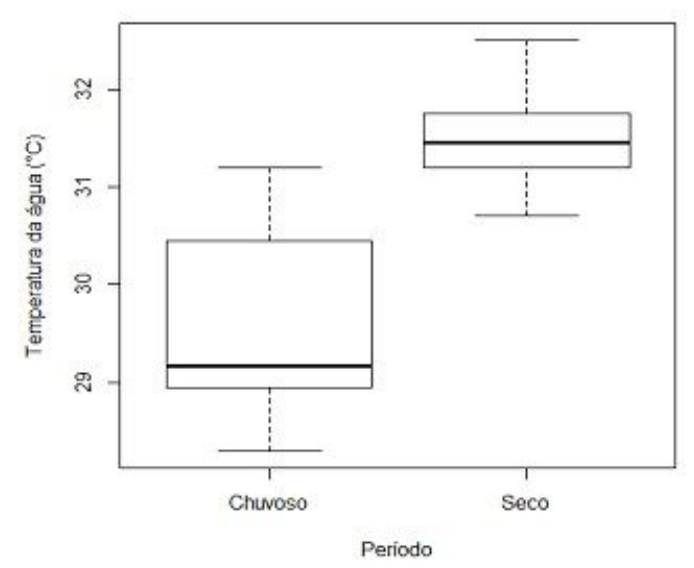

C)

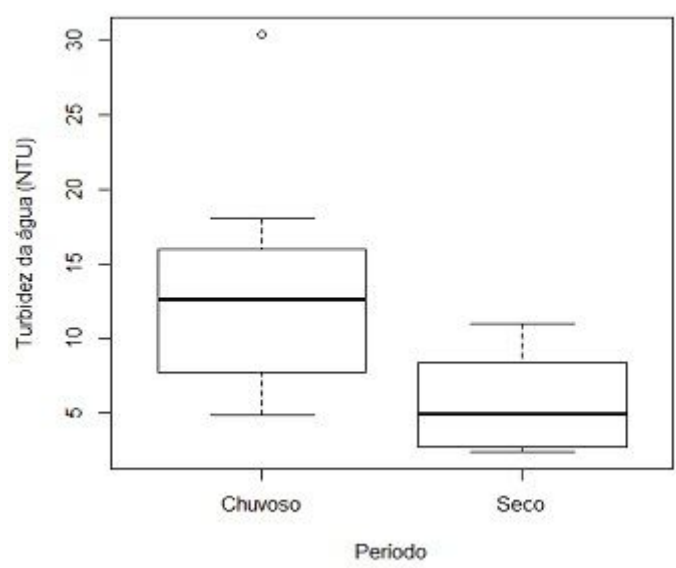

)
B)

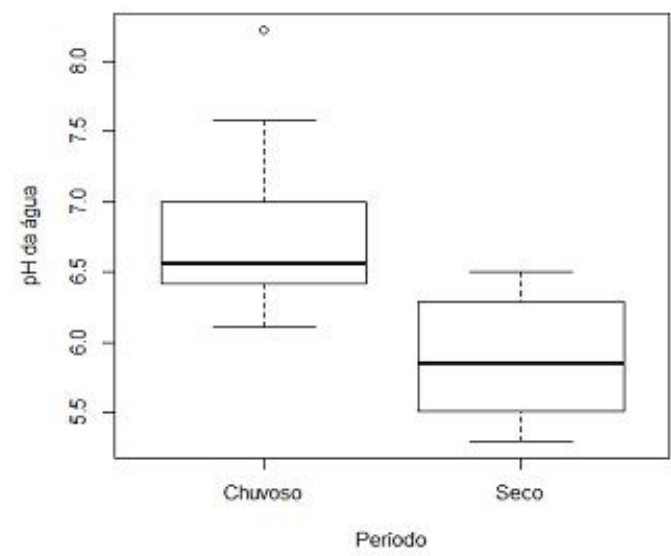

Em relação ao que foi registrado para OD em nosso estudo, com máxima de $8,3 \mathrm{mg} \mathrm{L}^{-1}$ no período chuvoso, o padrão observado foi oposto ao encontrado em outros reservatórios hidrelétricos amazônicos, como o de Samuel no rio Jamari (NASCIMENTO et al., 2020). Os valores máximos de OD ocorreram durante a seca e foram menores que os de Belo Monte, com concentração de $7,9 \mathrm{mg} \mathrm{L}^{-1}$. 
Possivelmente a diferença nas concentrações de OD entre os reservatórios de Samuel e Belo Monte, pode estar associada ao design dos reservatórios. Reservatórios a fio d'água como o de Belo Monte, mantém fluxo semelhante ao do rio em estado natural, enquanto reservatórios de acúmulo como o de Samuel tem padrão de fluxo mais lêntico e maior área alagada (EGRÉ; MILEWSKI, 2002). Portanto, o aumento das concentrações de OD observados durante o período chuvoso neste estudo, pode estar associado a maior ocorrência de eventos de turbulência que consequentemente podem aumentar o potencial de aeração da coluna d'água (BENNETT; RATHBUN, 1971).

As concentrações de $\mathrm{pH}$ foram mais elevadas durante o período chuvoso em contraste a seca. Foram registradas concentrações médias (Tabela 1), bem abaixo da concentração observada no reservatório de Tucuruí $(7,17)$ durante esse mesmo período (FREIRE et al., 2017). A acidificação do $\mathrm{pH}$ durante o período seco pode estar associada à disponibilidade de matéria orgânica no reservatório. O processamento de matéria orgânica pela comunidade microbiana em ambiente anóxico pode gerar elevadas concentrações de $\mathrm{CO}_{2}$ (BATTIN et al., 2008). Em ambiente aquático, o $\mathrm{CO}_{2}$ reage liberando ácido carbônico $\left(\mathrm{H}_{2} \mathrm{CO}_{3}\right)$, o que pode reduzir o pH (NEAL et al., 1998). A Supersaturação de $\mathrm{CO}_{2}$ foi registrada nos reservatórios da usina hidrelétrica de Belo Monte durante ambas as estações chuvosa e seca, especialmente em áreas posteriormente alagadas (ARAÚJO et al., 2019). Dessa forma, a produção de $\mathrm{CO}_{2}$ pelo reservatório pode estar associada à acidificação do $\mathrm{pH}$.

De acordo com a análise exploratória dos dados, o primeiro e segundo eixos da PCA explicaram $76 \%$ da variação dos dados físico-químicos (PC1: 0,48; PC2; $0,28)$ (Figura 3) da série histórica (Figura 4). As variáveis que melhor explicaram a variação dos dados foram turbidez e temperatura da água, onde os valores mais altos de temperatura são encontrados em locais com valores mais baixos de condutividade. Os dados pós barramento corroboram com os padrões encontrados com a PCA.

FIGURA 3 - PCA relativa à série histórica de dados físico-químicos no rio Xingu (A) e o critério de parada adotado (broken stick) (B) que define os eixos significativos para a análise (PC1: 2,28; PC2: 1,28).

A)

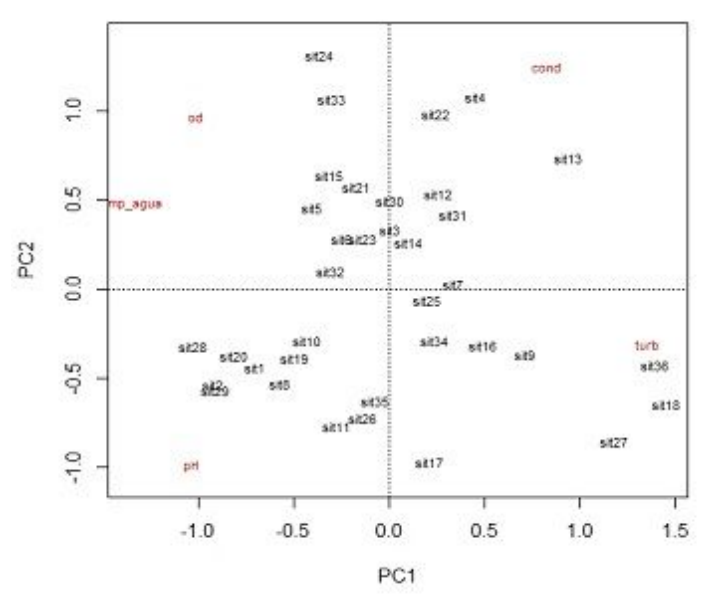

B)

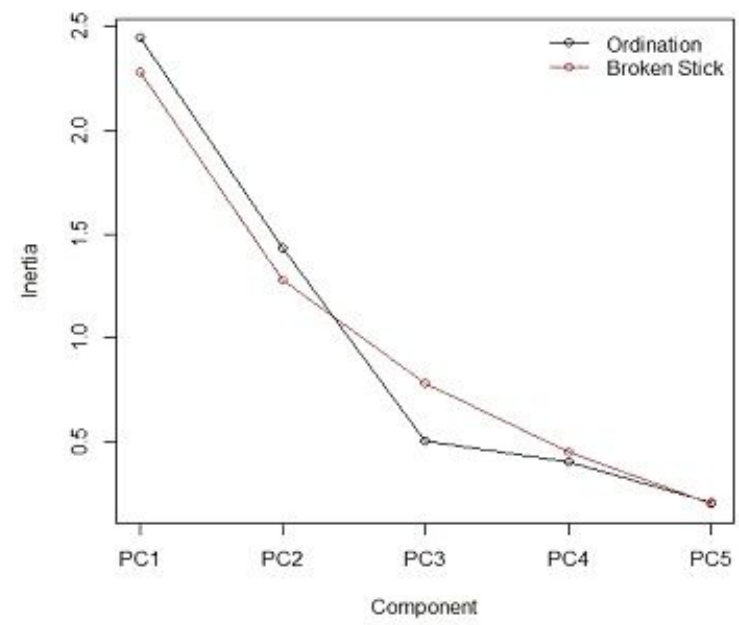


FIGURA 4 - Série histórica da média anual de temperatura da água (Temp), condutividade (Cond), $\mathrm{pH}$, turbidez (Turb) e oxigênio dissolvido (OD) durante o período de 2010 a 2020. As médias pré-represamento são relativas aos pontos (A) PR1, (B) PR2, (C) PR3 e (D) PR4.

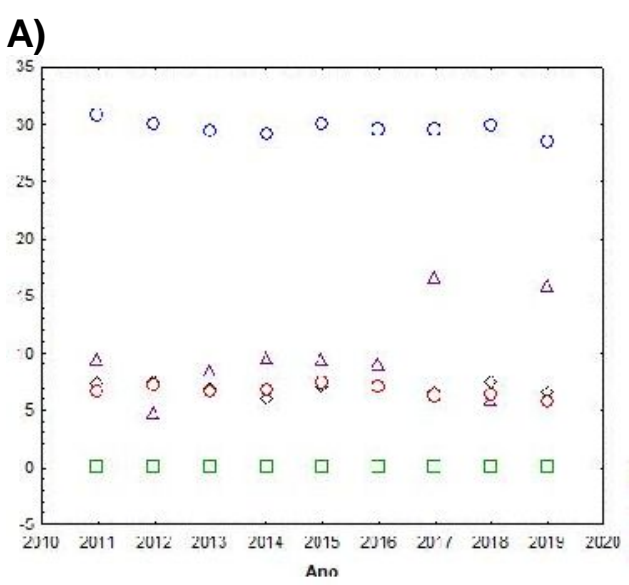

C)

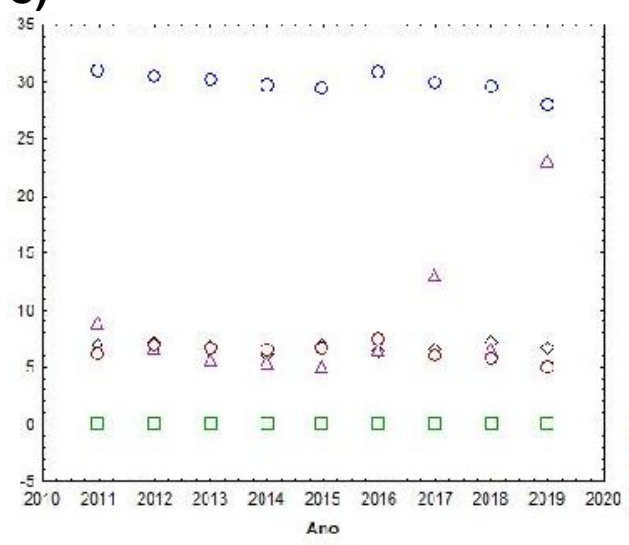

B)

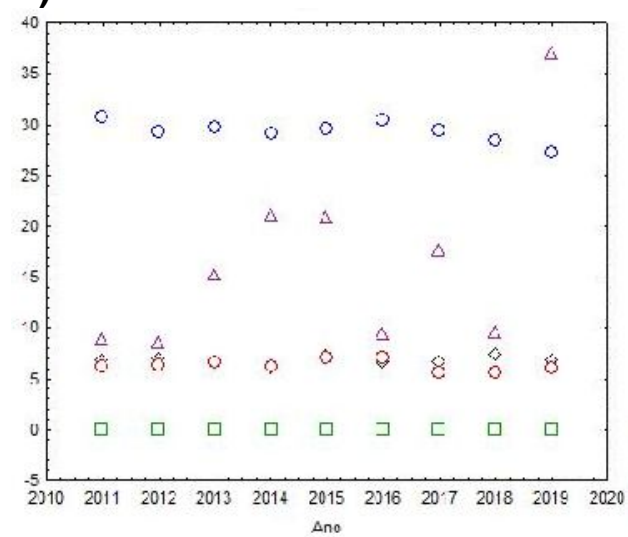

D)

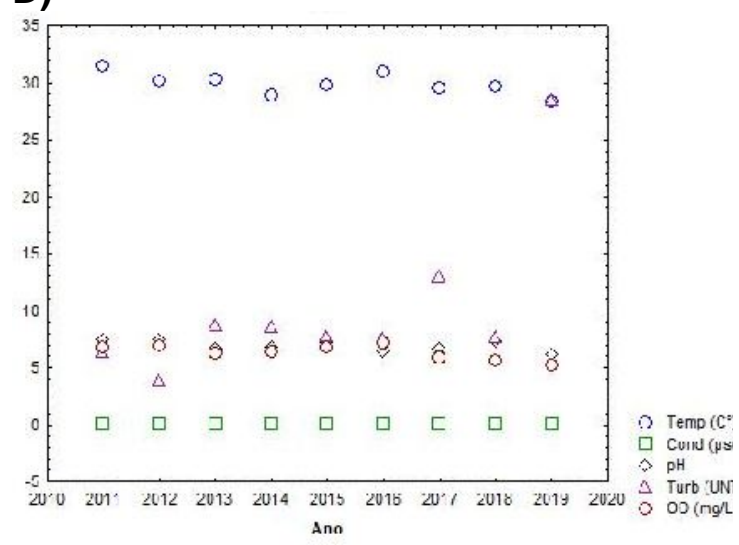

Para a temperatura da água, foi registrado valor máximo de $32,5^{\circ} \mathrm{C}$ durante 0 período seco e mínimo de $28,3^{\circ} \mathrm{C}$ no período chuvoso. O mesmo padrão foi observado nos valores de condutividade elétrica, com concentração máxima de 31 $\mu S \mathrm{~cm}^{-1}$ e mínima de $11 \mu \mathrm{S} \mathrm{cm} \mathrm{c}^{-1}$ (Tabela 1). Em comparação ao reservatório de Balbina, a temperatura da água tem variação similar (KASPER et al., 2014), o que já era esperado, tendo em vista que a região amazônica não apresenta diferenças climáticas muito expressivas. A temperatura da água pode ser afetada por diferentes processos naturais, como sazonalidade, latitude, longitude, vazão e profundidade (PERCEBON et al., 2005). Assim como também, atividades antrópicas como descarga de efluentes, represamento e desmatamento as margens dos rios ou igarapés. Dessa forma, se destaca a importância do conhecimento acerca da variação da temperatura da água nos ecossistemas aquáticos, considerando que mudanças bruscas de temperatura podem causar efeitos negativos em diversos processos que ocorrem nos ambientes aquáticos, como reações químicas e biológicas, que afetam a comunidade aquática (PELICICE et al., 2015). As alterações nas características limnológicas como temperatura, $\mathrm{pH}$ e turbidez, foi 
observada em função da estação seca e chuvosa em área de hidrelétrica (CHELLAPPA et al., 2009). As variáveis físico-químicas, apesar de se interrelacionarem, seguem as mudanças ambientais (ESTEVES, 1998). Dessa forma, o aumento da precipitação e consequentemente da turbidez, devido ao carreamento de sólidos das áreas adjacentes (AGOSTINHO et al., 2008), pode explicar o incremento da concentração de $\mathrm{pH}$.

Quando comparada a condutividade elétrica registrada no reservatório de Balbina, no rio Uatumã, foi observado um padrão oposto (KASPER et al., 2014). A máxima de condutividade elétrica $\left(21 \mu \mathrm{S} \mathrm{cm}^{-1}\right)$ observada durante a seca é bem inferior à concentração encontrada em nosso estudo. A condutividade elétrica elevada em Belo monte pode estar associada à idade do reservatório. Por se tratar de um reservatório recém construído, possui maior disponibilidade de matéria orgânica a ser deteriorada (GUÉRIN, et al., 2006). Nos dados pós-represamento a turbidez apresentou correlação negativa com temperatura da água $(p=-0,631, p<$ $0,001)$ (Figura 5-A) e positiva com $\mathrm{pH}(\mathrm{p}=0,508, \mathrm{p}=0,011)$ (Figura 5-B). Em um estudo anterior no rio Xingu, Rodrigues-Filho (2015) também relatou correlação positiva entre turbidez e temperatura. As outras variáveis analisadas não apresentaram correlação significativa neste estudo.

FIGURA 5: Correlações acima de 0,50 das variáveis nos períodos amostrados nas praias das margens do rio Xingu, Altamira-PA. A) Correlação negativa entre Temperatura e Turbidez; B) Correlação positiva entre pH e Turbidez.

A)

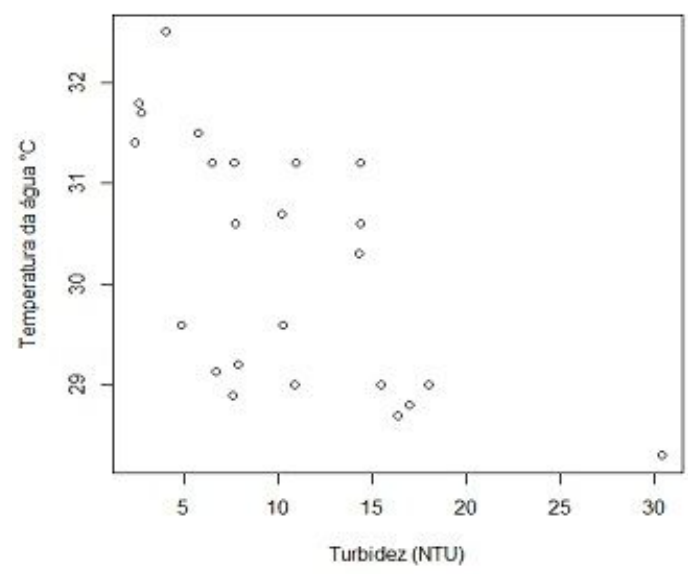

B)

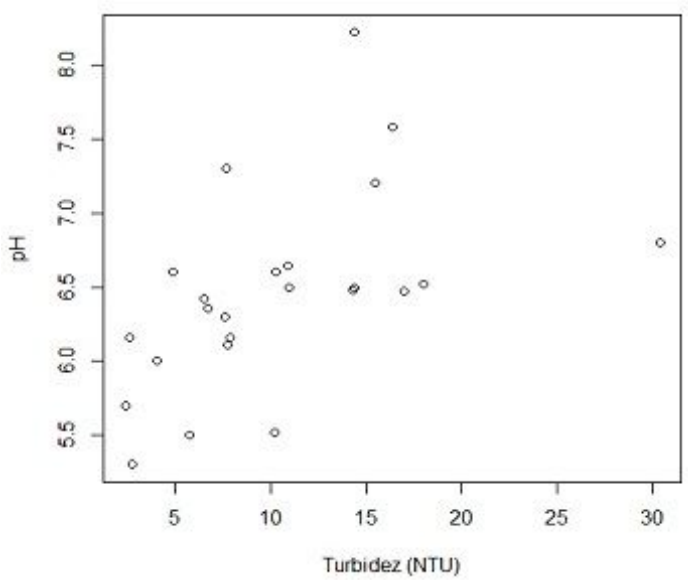

Padrões de temperatura da água afetam significativamente a atividade microbiana, que por sua vez pode influenciar as concentrações de OD (ESTEVES, 2011). Entretanto, não foi observado um efeito significativo da variação de temperatura sobre as concentrações de OD. A turbidez por outro lado, aparenta ter respondido melhor à mudança de temperatura durante cada período sazonal. Esse padrão já foi observado por Skorupa et al. (2017) analisando as variáveis limnológicas nas cabeceiras do Xingu. Porém, é possível que a correlação negativa registrada esteja associada à padrões de precipitação. É esperada turbidez mais elevada durante o período chuvoso devido a lixiviação dos solos e carreamento de sedimentos (ARCOVA; CICCO, 1999), assim como temperaturas mais baixas (ALVARES et al., 2013). Portanto, os resultados observados neste estudo apontam que as mudanças nas variações pós represamento tenham sido influenciadas pela sazonalidade. 


\section{CONCLUSÃO}

Não foi observada variação espacial nas variáveis limnológicas analisadas (Temperatura da água, Oxigênio Dissolvido, pH, Condutividade Elétrica e Turbidez). Contudo, houve alterações nos períodos seco e chuvoso em temperatura da água, $\mathrm{pH}$ e turbidez. Mesmo após o represamento, foi observado que o principal fator afetando as concentrações das variáveis limnológicas no rio Xingu foi a sazonalidade amazônica. Dessa forma, a caracterização limnológica considerando os períodos seco e chuvoso, deve ser considerada para o monitoramento dessas variáveis ao longo dos anos. Para avaliar mudanças nas condições hidrológicas das áreas afetadas por empreendimentos hidroelétricos.

\section{REFERÊNCIAS}

AGOSTINHO, A.; PELICICE, F.; GOMES, L. Dams and the fish fauna of the Neotropical region: impacts and management related to diversity and fisheries. Brazilian Journal of Biology, v. 68, n. 4 suppl, p. 1119-1132, nov/2008. Doi: $10.1590 /$ S1519-69842008000500019

ALMEIDA, R. M.; HAMILTON, S. K.; ROSI, E. J.; ARANTES, J. D.; BARROS, N. et al. Limnological effects of a large Amazonian run-of-river dam on the main river and drowned tributary valleys. Scientific Reports, v. 9, n. 1, p. 16846, dez/2019. Doi: 10.1038/s41598-019-53060-1

ALVARES, C. A.; STAPE, J. L.; SENTELHAS, P. C.; MORAES-GONÇALVES, J. L.; SPAROVEK, G. Köppen's climate classification map for Brazil. Meteorologische Zeitschrift, v. 22, n. 6, p. 711-728, 1 dez/2013. Doi: 10.1127 / 0941-2948 / 2013/0507

ANDERSON, D.; MOGGRIDGE, H.; WARREN, P.; SHUCKSMITH, J. The impacts of 'run-of-river' hydropower on the physical and ecological condition of rivers. Water and Environment Journal, v. 29, n. 2, p. 268-276, jun/2015. Doi: 10.1111/wej.12101

ARAÚJO, K. R.; SAWAKUCHI, H. O.; BERTASSOLI-JR, D. J.; SAWAKUCHI, A. O.; DIAS-Silva, K. et al. Carbon dioxide (CO2) concentrations and emission in the newly constructed Belo Monte hydropower complex in the Xingu River, Amazonia. Biogeosciences, v. 16, n. 18, p. 3527-3542, 18 set/2019. Doi: 10.5194/bg-16-35272019

ARCOVA, F. C. S.; CICCO, V. DE. Qualidade da água de microbacias com diferentes usos do solo na região de Cunha, Estado de São Paulo. Scientia Forestalis , p. 125-134, 1999. Disponivel em: < https://www.ipef.br/publicacoes/scientia/nr56/cap09.pdf >.

BATALHA, S. S. A.; MARTORANO, L. G.; BIASE, A. G.; MORALES, G. P.; PONTES, A. N.; SANTOS, L. Condições físico-químicas e biológicas em águas superficiais do Rio Tapajós e a conservação de Floresta Nacional na Amazônia, Brasil. Ambiente e Agua - An Interdisciplinary Journal of Applied Science, v. 9, n. 4, 17 out/ 2014. Doi: 10.4136/ambi-agua.1304bata

BATTIN, T. J.; KAPLAN, L. A.; FINDLAY, S.; HOPKINSON, C. S.; MARTI, E. et al. 
Biophysical controls on organic carbon fluxes in fluvial networks. Nat. Geosci., 2, 595-595, 2008. Doi: 10.1038/ngeo101

BENNETT, J. P., RATHBUN, R. E. Reaeration in open-channel flow. Department of the Interior US, Geological Survey, 1971.

BRASIL. Resolução CONAMA 357, de 17 de março de 2005. Conselho Nacional de Meio Ambiente. Disponível em: <www.mma.gov.br/port/conama/res/res05/res35705.pdf>.

CASTELLO, L.; MACEDO, M. N. Large-scale degradation of Amazonian freshwater ecosystems. Global Change Biology, v. 22, n. 3, p. 990-1007, mar/2016. Doi: $10.1111 / \mathrm{gcb} .13173$

CHANUdET, V., GUÉdANT, P., RODE, W., GODON, A., GUÉRIN, F., et al. Evolution of the physico-chemical water quality in the Nam Theun 2 Reservoir and downstream rivers for the first 5 years after impoundment. Hydroécologie Appliquée, 19, 27-61, 2016. Doi: 10.1051/hydro/2015001

CHELLAPPA, N.T.; CHELLAPPA, T.; CÂAMARA, F.R.A.; ROCHA, O.; CHELLAPPA, $S$. Impact of stress and disturbance factors on the phytoplankton communities in Northeastern Brazil reservoir. Limnologica 39:273-282., 2009. Doi: 10.1016/j.limno.2009.06.006

CONCEIÇÃO, L. P., DE JESUS AFFE, H. M., DA SILVA, D. M. L., DE CASTRO NUNES, J. M. Spatio-temporal variation of the phytoplankton community in a tropical estuarine gradient, under the influence of river damming. Regional Studies in Marine Science, 43, 101642, 2021. Doi: 10.1016/j.rsma.2021.101642

EGRÉ, D.; MILEWSKI, J. C. The diversity of hydropower projects. Energy Policy, 30(14), 1225-1230, 2002. Doi: 10.1016/S0301-4215(02)00083-6

\section{ELETROBRÁS. AAl-Avaliação Ambiental Integrada Aproveitamentos Hidrelétricos da Bacia Hidrográfica do Rio Xingu Volume II Ministério de Minas e Energia. São Paulo: 2009. Disponível em: $<$ https://eletrobras.com/pt/AreasdeAtuacao/geracao/belo_monte/AAI-Xingu-Volume- II.pdf>.}

ESTEVES, F. DE A. Fundamentos de limnologia. 2. ed. Rio de Janeiro: Interciência, 1998.

ESTEVES, F. DE A. Fundamentos de Limnologia. 3. ed. Rio de Janeiro: Interciência, 2011.

FREIRE, J. C. A.; HAUSER-DAVIS, R. A.; DA COSTA LOBATO, T.; DE MORAIS, J. M.; DE OLIVEIRA, T. F.; et al. Influence of the Amazon Hydrological Regime on Eutrophication Indicators of a Hydroelectric Power Plant Reservoir. Bulletin of environmental contamination and toxicology, 98(5), 677-681., 2017. Doi: https://doi.org/10.1007/s00128-017-2030-9 
GUÉRIN, F., ABRIL, G., RICHARD, S., BURBAN, B., REYNOUARD, C. et al. Methane and carbon dioxide emissions from tropical reservoirs: Significance of downstream rivers, Geophys. Research Letters, 33, 1-6, 2006. Doi: 10.1029/2006GL027929

IBGE - Instituto Brasileiro de Geografia e Estatistica - Censo demográfico 2010. Disponível em: <https://ftp.ibge.gov.br/Censos/Censo_Demografico_2010/Resultados_Diario_Oficial _da_Uniao_2010_11_04/PA2010.pdf>.

INMET- Instituto Nacional de Metereologia - Banco de dados metereológico . Disponível em:

br/assuntos/inmet?r=bdmep/bdmep $>$.

$<$ https://www.gov.br/agricultura/pt-

KASPER, D.; FORSBERG, B. R.; AMARAL, J. H.; LEITÃO, R. P.; PY-DANIEL, S. S.; et al. Reservoir stratification affects methylmercury levels in river water, plankton, and fish downstream from Balbina hydroelectric dam, Amazonas, Brazil. Environmental science \& technology, 48(2), 1032-1040, 2014. Doi: 10.1021/es4042644

KOEPPEN, W. Climatologia: con un estudio de los climas de la tierra. Buenos Aires: Fondo de Cultura Econômica, 1984.

LAURANCE, W. F.; COCHRANE, M. A.; BERGEN, S.; FEARNSIDE, P. M.; DELAMÔNICA, P. et al. ENVIRONMENT: The Future of the Brazilian Amazon. Science, v. 291, n. 5503, p. 438-439, 19 jan. 2001. Doi: 10.1126/science.291.5503.438

LEGENDRE, P.; LEGENDRE, L. Numerical ecology. 3. ed. Amsterdam: Elsevier, 2012.

LING, T. Y.; GERUNSIN, N.; SOO, C. L.; NYANTI, L., SIM, S. F. et al. Seasonal changes and spatial variation in water quality of a large young tropical reservoir and its downstream river. Journal of Chemistry, 2017. Doi: 10.1155/2017/8153246

MULLIGAN, M.; VAN SOESBERGEN, A.; SÁENZ, L. GOODD, a global dataset of more than 38,000 georeferenced dams. Scientific Data, 7:1-8., 2020. Doi: $10.1038 / \mathrm{s} 41597-020-0362-5$

NASCIMENTO, E. L.; MIYAI, R .K.; DE OLIVEIRA GOMES, J.; DE ALMEIDA, R.; DE CARVALHO, D. P. et al. Dynamics of mercury in the plankton of a hydroelectric reservoir, Western Amazon. Environ Monit Assess 192, 647, 2020. Doi: 10.1007/s10661-020-08600-y

NEAL, C.; HOUSE, W.; JARVIE, H.; THERALL, A. The significance of dissolved carbon dioxide in major lowland rivers entering the North Sea. Science of The Total Environment, v. 210-211, p. 187-203, 24 mar. 1998. Doi: 10.1016/S00489697(98)00012-6

NEPSTAD, D.; MCGRATH, D.; STICKLER, C.; ALENCAR, A.; AZEVEDO, A. et al. Slowing Amazon deforestation through public policy and interventions in beef and 
soy supply chains. Science, v. 344, n. 6188, p. 1118-1123, 6 jun. 2014. Doi: 10.1126/science. 1248525

NOGUEIRA, M. G.; PERBICHE-NEVES, G.; NALIATO, D. O.; CASANOVA, S. M. C.; DEBASTIANI-JÚNIOR, J. R. et al. Limnology and water quality in La Plata basin (South America) - Spatial patterns and major stressors. Ecological Indicators, v. 120, p. 106968, jan. 2021. Doi: https://doi.org/10.1016/j.ecolind.2020.106968

OKSANEN, J. BLANCHET, F.; FRIENDLY, G.; KINDT, M.; LEGENDRE, P. R. et al. vegan: Community Ecology Package. Disponível em: <https://cran.rproject.org/web/packages/vegan/index.html>.

PELICICE, F. M.; POMPEU, P. S.; AGOSTINHO, A. A. Large reservoirs as ecological barriers to downstream movements of Neotropical migratory fish. Fish and Fisheries, v. 16, n. 4, p. 697-715, dez/2015. Doi: 10.1111/faf.12089

PERCEBON, C.M.; BITTENCOURT, A.V.L.; FILHO, E. F. da R. Diagnóstico da temperatura das águas dos principais rios de Blumenau, SC. Boletim Paranaense de Geociências, n. 56, p. 7-19. Editora UFPR. 2005. Disponivel em: <https://core.ac.uk/download/pdf/328070554.pdf>.

POFF, N. L.; WARD, J. V. Implications of Streamflow Variability and Predictability for Lotic Community Structure: A Regional Analysis of Streamflow Patterns. Canadian Journal of Fisheries and Aquatic Sciences, v. 46, n. 10, p. 1805-1818, 1 out/. 1989. Doi: $10.1139 / f 89-228$

R CORE TEAM. R: A language and environment for statistical computing. $\mathbf{R}$ Foundation for Statistical Computing. Disponível em: <https://www.r-project.org/>.

RíOS-VILLAMIZAR, E. A.; PIEDADE, M. T. F.; JUNK, W. J.; WAICHMAN, A. V. Surface water quality and deforestation of the Purus river basin, Brazilian Amazon. International Aquatic Research, v. 9, n. 1, p. 81-88, 2 mar/2017. Doi: 10.1007/s40071-016-0150-1

RODRIGUES-FILHO, J. L.; ABE, D. S.; GATTI-JUNIOR, P.; MEDEIROS, G. R.; DEGANI, R. M. et al. Padrões espaciais de qualidade da água na Bacia do Rio Xingu (Amazônia) antes do represamento da barragem de Belo Monte. Revista Brasileira de Biologia, 75 (3, Suplemento 1), 34-46, 2015. Doi: https://doi.org/10.1590/15196984.02914bm

SANTOS, C. A.; LIMA, A. M. M.; FRANCO, V. S.; ARAUJO, I. B.; MENEZES, J. F. G. et al.; Distribuição Espacial da Precipitação na Bacia Hidrográfica do Rio Xingu. Nucleus, v. 13, n. 2, p. 223-230, 30 out. 2016. Doi: 10.3738/1982.2278.1630

SHELDON, F.; PETERSON, E. E.; BOONE, Ed. L.; SIPPEL, S.; BUNN, S. E. et al. Identifying the spatial scale of land use that most strongly influences overall river ecosystem health score. Ecological Applications, v. 22, n. 8, p. 2188-2203, dez/. 2012. Doi: $10.1890 / 11-1792.1$

SILVÉRIO, D. V.; BRANDO, P. M.; MACEDO, M. N.; BECK, P. S. A.; 
BUSTAMANTE, M. et al. Agricultural expansion dominates climate changes in southeastern Amazonia: the overlooked non-GHG forcing. Environmental Research Letters, v. 10, n. 10, p. 104015, 1 out/2015. Doi: 10.1088/1748-9326/10/10/104015

SIOLI, H. The Amazon and its main affluents: Hydrography, morphology of the river courses, and river types. In: The Amazon: limnology and landscape ecology of a mighty tropical river and its basin. Dordrecht: Springer Science \& Business Media, p. 127-165. 1984.

SKOLE, D. L.; CHOMENTOWSKI, W. H.; SALAS, W. A.; NOBRE, A. D. Physical and Human Dimensions of Deforestation in Amazonia. BioScience, v. 44, n. 5, p. 314322, mai/1994. Doi: $10.2307 / 1312381$

SKORUPA, L. A.; COSTA, U. M. P.; CUNHA, P.; ROSSETE, A. N.; FIDALGO, E. C. C.; SIMÕES, M.; et al.,; Monitoramento da qualidade da água na bacia hidrográfica do rio Suiá-Miçu. Embrapa: 2017. Disponivel em: <https://www.embrapa.br/busca-de-publicacoes/-

/publicacao/1080466/monitoramento-da-qualidade-da-agua-na-bacia-hidrograficado-rio-suia-micu>.

ST. LOUIS, V.L.; KELLY, C.A.; DUCHEMIN, E.; RUDD, J. W.M.; ROSENBERG, DAVID M. Reservoir surfaces as sources of greenhouse gases to the atmosphere: a global estimate. BioScience, 50:766-775., 2000. Doi: 10.1641/00063568(2000)050[0766:RSASOG]2.0.CO;2

TRÉPANIER, S.; RODRíGUEZ, M. A.; MAGNAN, P. Spawning migrations in landlocked Atlantic salmon: Time series modelling of river discharge and water temperature effects. Journal of Fish Biology, v. 48, n. 5, p. 925-936, 1 mai/1996. Doi: 10.1111/j.1095-8649.1996.tb01487.x

TUNDISI, J. G. Exploração do potencial hidrelétrico da Amazônia. Estud. av., São Paulo , v. 21, n. 59, p. 109-117, abr/2007. Doi: 10.1590/S010340142007000100009.

VOZA, D.; VUKOVIC, M.; TAKIC, L.; NIKOLIC, D.; MLADENOVIC-RANISAVLJEVIC, I. Application of multivariate statistical techniques in the water quality assessment of Danube river, Serbia. Archives of Environmental Protection, v. 41, n. 4, p. 96103, 1 dez/2015. Doi: 10.1515/aep-2015-0044

ZAR, J. H. Biostatistical analysis. 5. ed. New Jersey: Pearson, 2010. 